PROCEEDINGS OF THE

AMERICAN MATHEMATICAL SOCIETY

Volume 139, Number 11, November 2011, Pages 4087-4095

S 0002-9939(2011)10883-9

Article electronically published on March 25, 2011

\title{
ON CONVERGENCE OF THE PROXIMAL POINT ALGORITHM IN BANACH SPACES
}

\author{
SHIN-YA MATSUSHITA AND LI XU
}

(Communicated by Thomas Schlumprecht)

\begin{abstract}
In this paper, we give a sufficient condition which guarantees that the sequence generated by the proximal point algorithm terminates after a finite number of iterations.
\end{abstract}

\section{INTRODUCTION}

Let $E$ be a Banach space and $E^{*}$ be its dual space. We consider the problem of finding a point $u \in E$ such that

$$
0 \in T(u)
$$

where $T$ is a maximal monotone operator from $E$ to $2^{E^{*}}$. The problem of finding a solution of (1.1) has interesting interpretations in various fields. For instance, convex minimization problems can be written in this form by setting $T=\partial f$, where $\partial f$ is the subdifferential mapping of a convex function $f$. Other problems such as saddle point problems, variational inequalities, and complementarity problems can also be written in (1.1) (see, e.g., 3, 4, 26, 27, 30). Until now, a variety of methods for solving (1.1) has been proposed and investigated (see, e.g., [26, 7, 23, 20, 17, 18, 29, 5, 16, 19]). Among them, the proximal point algorithm, which was first proposed by Martinet [22, is known for its theoretically nice convergence properties. The proximal point algorithm generates, for any initial point $x_{1} \in E$, a sequence $\left\{x_{n}\right\}$ converging to a solution of (1.1) by the iterative scheme:

$$
x_{n+1}=J_{r_{n}}\left(x_{n}\right)(n=1,2, \ldots),
$$

where $J_{r_{n}}: E \rightarrow E$ is the mapping defined by $\left(J+r_{n} T\right)^{-1} J, J$ is the duality mapping on $E$ and $\left\{r_{n}\right\}$ is a positive sequence. In this paper, we are interested in the conditions that ensure that the sequence $\left\{x_{n}\right\}$ generated by (1.2) can obtain an exact solution of (1.1) in a finite number of iterations.

It is well known that the proximal point algorithm has nice convergence properties. In a Hilbert space setting, Rockafellar [26] proved that if $\liminf _{n \rightarrow \infty} r_{n}>0$ and (1.1) has at least one solution, then $\left\{x_{n}\right\}$ converges weakly to a solution of

Received by the editors September 6, 2010.

2010 Mathematics Subject Classification. Primary 65K10, 90C25; Secondary 47J25, 47H05, $47 \mathrm{H} 04$.

Key words and phrases. Proximal point algorithm, finite termination of algorithm, maximal monotone operator, paramonotone operator, Banach space, weak sharp minima.

The first author was supported by Grant-in-Aid for Young Scientists (B) No. 20740084, the Ministry of Education, Culture, Sports, Science and Technology, Japan.

(C)2011 American Mathematical Society Reverts to public domain 28 years from publication 
(1.1). Further generalizations of Rockafellar's result were discussed in [17, 16. However, some examples of sequences generated by (1.2) that converge weakly but not strongly are known [14, 6]. That is, to prove finite termination of (1.2) in infinite dimensional spaces, some additional assumptions are needed. In a Banach space setting, Kassay [17] proved that if there exists $u \in E$ such that $0 \in \operatorname{int} T(u)$, then $\left\{x_{n}\right\}$ terminates after a finite number of iterations. It should be noted that condition $0 \in \operatorname{int} T(u)$ is rather strong because it implies that $u$ is the unique solution of (1.1). On the other hand, in a finite dimensional setting, Ferris [13. considered the finite termination of the proximal point algorithm for convex programming problems. He gave a condition which is closely related to the notion of weak sharp minima [8, 9 ] and applied it to obtain finite convergence results. It is known that the notion of weak sharp minima has many important applications in sensitivity analysis of convex programming problems and complementarity problems, and in the convergence analysis of some descent methods (see, e.g., [21, 13, 8, 9, 31). It should be noted that the proof of Ferris' results relies on properties of the subdifferential mapping of convex functions.

The purpose of this paper is to propose a sufficient condition that ensures finite termination of the sequence generated by (1.2) in Banach spaces. For this purpose, a generalization of Ferris' condition which can be applied to set-valued operators is introduced and investigated. Our condition does not require that the solution set be a singleton or even a bounded set. Applying this condition, we prove two lemmas which are important for the proof of the main results. Then we establish two finite convergence results of the proximal point algorithm. Finally, we apply our convergence results to the convex minimization problem. These results include the corresponding results in [21, 13] as special cases.

\section{BASIC DEFINITIONS AND PRELIMINARIES}

Let $\mathbb{N}$ and $\mathbb{R}$ denote the sets of positive integers and real numbers, respectively. Let $E$ be a real Banach space with norm $\|\cdot\|$ and let $E^{*}$ be the dual space of $E$. By $\left\langle x, x^{*}\right\rangle$ we denote the value of the continuous linear functional $x^{*} \in E^{*}$ at $x \in E$. $B(0, \epsilon)=\{y \in E:\|y\| \leq \epsilon\}$ and $B^{*}(0, \epsilon)=\left\{y^{*} \in E^{*}:\left\|y^{*}\right\| \leq \epsilon\right\}$ are the closed balls of $E$ and $E^{*}$ with radius $\epsilon>0$, respectively. The duality mapping $J$ from $E$ into $E^{*}$ is defined by

$$
J(x)=\left\{x^{*} \in E^{*}:\left\langle x, x^{*}\right\rangle=\|x\|^{2}=\left\|x^{*}\right\|^{2}\right\}
$$

for all $x \in E$. A Banach space $E$ is said to be

(i) smooth if the limit

$$
\lim _{t \rightarrow 0} \frac{\|x+t y\|-\|x\|}{t}
$$

exists for all $x, y \in S(E)=\{z \in E:\|z\|=1\}$;

(ii) uniformly smooth if the limit (2.2) exists uniformly in $x, y \in S(E)$;

(iii) strictly convex if $\left\|\frac{x+y}{2}\right\|<1$ whenever $x, y \in S(E)$ with $x \neq y$;

(iv) uniformly convex if for each $\epsilon \in(0,2]$, there exists $\delta>0$ such that $\left\|\frac{x+y}{2}\right\| \leq$ $1-\delta$ whenever $x, y \in S(E)$ with $\|x-y\| \geq \epsilon$.

We list the following useful properties of the duality mapping:

1. If $E$ is reflexive, then $J$ is surjective;

2. if $E$ is smooth, then $J$ is single-valued; 
3. if $E$ is uniformly smooth, then $J$ is uniformly norm-to-norm continuous on each bounded subset of $E$;

see [11, 4, 30] for more details. For example, if $p$ is a real number such that $1<p<\infty$, then the sequence space $l^{p}$ and the Lebesgue space $L^{p}$ are uniformly convex and uniformly smooth.

For a given set $C$ in $E$, we denote the interior of $C$ by int $C$. The normal cone to $C$ at $x$ is defined by

$$
N_{C}(x)=\left\{x^{*} \in E^{*}:\left\langle x^{*}, y-x\right\rangle \leq 0 \text { for all } y \in C\right\} .
$$

Let $C$ be a nonempty, closed and convex subset of a smooth, strictly convex and reflexive Banach space $E$. Then for each $x \in E$, there corresponds a unique element $x_{0} \in C$ (denoted by $\left.P_{C}(x)\right)$ such that $\left\|x_{0}-x\right\|=\min _{y \in C}\|y-x\| . P_{C}(x)$ satisfies

$$
\left\langle y-P_{C}(x), J\left(x-P_{C}(x)\right)\right\rangle \leq 0 \text { for all } y \in C ;
$$

see 1, 30, for more details.

Consider a set-valued operator $T: E \rightarrow 2^{E^{*}}$. Its domain $D(T)$, range $R(T)$ and graph $G(T)$ are defined by $D(T)=\{z \in E: T(z) \neq \emptyset\}, R(T)=\bigcup\left\{T(z) \in 2^{E^{*}}\right.$ : $z \in D(T)\}$ and $G(T)=\left\{\left(x, x^{*}\right) \in E \times E^{*}: x^{*} \in T(x)\right\}$, respectively. The inverse $T^{-1}: E^{*} \rightarrow 2^{E}$ of $T$ is defined by $T^{-1}\left(x^{*}\right)=\left\{x \in E: x^{*} \in T(x)\right\}$ for $x^{*} \in R(T)$ and $T^{-1}\left(x^{*}\right)=\emptyset$, otherwise. $T$ is said to be

(i) monotone if

$$
\left\langle x-y, x^{*}-y^{*}\right\rangle \geq 0 \text { for all }\left(x, x^{*}\right),\left(y, y^{*}\right) \in G(T) ;
$$

(ii) paramonotone [10, 12 if it is monotone and

$$
\left\langle x-y, x^{*}-y^{*}\right\rangle=0 \text { with }\left(x, x^{*}\right),\left(y, y^{*}\right) \in G(T) \Rightarrow x^{*} \in T(y) \text { and } y^{*} \in T(x) \text {. }
$$

A monotone operator $T$ is said to be maximal monotone if

$$
U: E \rightarrow 2^{E^{*}} \text { is monotone and } G(T) \subset G(U) \Rightarrow T=U .
$$

When $T$ is maximal monotone, the solution set $T^{-1}(0)$ of (1.1) is closed and convex. We know that the subdifferential of a proper, convex and lower semicontinuous function is maximal monotone; see for example [24, 2]. Some examples of paramonotone operators were discussed in 10, 12. The following result is an extension of Iusem's result [12, Proposition 2.2] to more general Banach spaces, which also shows an important example of a paramonotone operator.

Proposition 2.1. Let $f: E \rightarrow \overline{\mathbb{R}}=\mathbb{R} \cup\{\infty\}$ be a proper, convex and lower semicontinuous function. Then $\partial f: E \rightarrow 2^{E^{*}}$ is paramonotone, where $\partial f$ is the subdifferential mapping defined by

$$
\partial f(x)=\left\{x^{*} \in E^{*}: f(y) \geq f(x)+\left\langle y-x, x^{*}\right\rangle \text { for all } y \in E\right\}
$$

for all $x \in E$.

Proof. Assume that $\left\langle x-y, x^{*}-y^{*}\right\rangle=0$ with $x^{*} \in \partial f(x)$ and $y^{*} \in \partial f(y)$. Define $f_{0}: E \rightarrow \overline{\mathbb{R}}$

$$
f_{0}(z)=f(z)+\left\langle x-z, x^{*}\right\rangle .
$$

From (2.7), we have $\partial f_{0}(z)=\partial f(z)-x^{*}$. By $x^{*} \in \partial f(x)$ and $y^{*} \in \partial f(y)$,

$$
f(x)-f(y) \leq\left\langle x-y, x^{*}\right\rangle=\left\langle x-y, y^{*}\right\rangle \leq f(x)-f(y) .
$$


This implies that $f(x)=f(y)+\left\langle x-y, x^{*}\right\rangle=f_{0}(y)$. It follows that $f_{0}(x)=f(x)=$ $f_{0}(y)$. Since $0 \in \partial f(x)-x^{*}=\partial f_{0}(x), x$ is an unrestricted minimizer of $f_{0}$, so that $y$ is also an unrestricted minimizer of $f_{0}$. It follows that

$$
0 \in \partial f_{0}(y)=\partial f(y)-x^{*} \text {. }
$$

Thus, we obtain $x^{*} \in \partial f(y)$. Similarly, we can show that $y^{*} \in \partial f(x)$.

Throughout this paper we assume paramonotonicity of $T$.

\section{LEMMAS}

In this section, we propose a generalization of Ferris' condition in a Banach space and obtain two lemmas which are important for the ensuing theorems.

In a finite dimensional setting, Ferris [13] considered the following condition: There exists $\alpha>0$ such that

$$
B(0, \alpha) \cap\left(\bigcup_{x \in S} N_{S}(x)\right) \subset \bigcup_{x \in S} \partial\left(f+i_{C}\right)(x),
$$

where $f: \mathbb{R}^{n} \rightarrow \overline{\mathbb{R}}$ is a proper, convex and lower semicontinuous function, $C$ is a closed convex subset of $\mathbb{R}^{n}, i_{C}: \mathbb{R}^{n} \rightarrow \overline{\mathbb{R}}$ is the indicator function of $C$ defined by $i_{C}(x)=0$ for $x \in C$ and $i_{C}(x)=\infty$, otherwise, and $S$ is the solution set of a convex programming problem, i.e., $S=\left\{u \in C: f(u)=\min _{x \in C} f(x)\right\}$. It has been shown [8. Theorem 2.2] that condition (3.1) holds if and only if the set $S$ is a set of weak sharp minima 8, 9 for the function $f$ with modulus $\alpha$. There are many examples of convex functions that have a set of weak sharp minima; see for example [21, 8. By applying (3.1), Ferris [13, proved finite convergence results of a proximal point algorithm for convex programming problems.

Now, we consider the condition

$$
B^{*}(0, \alpha) \cap\left(\bigcup_{x \in \bar{S}} N_{\bar{S}}(x)\right) \subset \bigcup_{x \in \bar{S}} T(x),
$$

where $T: E \rightarrow 2^{E^{*}}$ is a set-valued operator and $\bar{S}=T^{-1}(0)$. In a Banach space setting, the subdifferential $\partial\left(f+i_{C}\right)$ of $f+i_{C}$ is a set-valued operator from $E$ to $2^{E^{*}}$ and $S=\partial\left(f+i_{C}\right)^{-1}(0)$. Thus, (3.2) can be regarded as a natural extension of (3.1). We next consider the relationship between condition $0 \in \operatorname{int} T(u)$ and condition (3.2). For a monotone operator, we obtain the following result.

Proposition 3.1. Let $T: E \rightarrow 2^{E^{*}}$ be a monotone operator with $\bar{S} \neq \emptyset$. Then $T(x) \subset N_{\bar{S}}(x)$ for all $x \in E$.

Proof. Let $x \in D(T)$ and $x^{*} \in T(x)$. Since $T$ is monotone, $\left\langle x-z, x^{*}\right\rangle \geq 0$ for all $z \in \bar{S}$. From (2.3), we have that $x^{*} \in N_{\bar{S}}(x)$.

Remark 3.2. We can prove that if there exists $u \in E$ such that $0 \in \operatorname{int} T(u)$, then (3.2) holds. In fact, since there exists $\delta>0$ such that $B^{*}(0, \delta) \subset T(u)$, it follows from Proposition 3.1 that $\bigcup_{x \in \bar{S}} T(x) \subset \bigcup_{x \in \bar{S}} N_{\bar{S}}(x)$. This implies that $B^{*}(0, \delta) \cap\left(\bigcup_{x \in \bar{S}} N_{\bar{S}}(x)\right) \subset \bigcup_{x \in \bar{S}} T(x)$. Therefore, condition (3.2) is much weaker than $0 \in \operatorname{int} T(u)$. Moreover, condition (3.2) does not require that the solution set be a singleton or even a bounded set. 
We will apply condition (3.2) to obtain finite convergence results of the proximal point algorithm. We first show the following two lemmas, which are an extension of Ferris' results [13, Lemmas 4 and 5] from the finite dimensional space to the Banach space. The key difference of our approach to Ferris' is that we do not require that $T$ be a subdifferential mapping $\partial\left(f+i_{C}\right)$ of $f+i_{C}$ defined on $\mathbb{R}^{n}$.

Lemma 3.3. Let $E$ be a smooth, strictly convex and reflexive Banach space, let $T$ : $E \rightarrow 2^{E^{*}}$ be a maximal monotone and paramonotone operator with $\bar{S}=T^{-1}(0) \neq \emptyset$, and let $z \in E$. For any $\lambda>0$, let $y^{*}=\lambda J\left(z-P_{\bar{S}}(z)\right)$. If there exists $w \in \bar{S}$ such that $y^{*} \in T(w)$, then $y^{*} \in T\left(P_{\bar{S}}(z)\right)$.

Proof. Since $T$ is monotone and using (2.4), we have

$$
\begin{aligned}
0 & \leq\left\langle w-P_{\bar{S}}(z), y^{*}-0\right\rangle \\
& =\left\langle w-P_{\bar{S}}(z), \lambda J\left(z-P_{\bar{S}}(z)\right)\right\rangle \\
& \leq 0 .
\end{aligned}
$$

Since $T$ is a paramonotone operator, we have $y^{*} \in T\left(P_{\bar{S}}(z)\right)$.

Remark 3.4. The proof of Ferris' result [13, Lemma 4] relies on properties of the subdifferential mapping of the convex functions.

Lemma 3.5. Let $E$ be a smooth, strictly convex and reflexive Banach space and let $T$ be a maximal monotone and paramonotone operator. Suppose that there exists $\alpha>0$ such that condition (3.2) holds. If $\left\|w^{*}\right\|<\alpha$ and $w^{*} \in T(z)$, then $z \in \bar{S}$.

Proof. Let us assume that $z \notin \bar{S}$. Define

$$
y^{*}=\alpha \frac{J\left(z-P_{\bar{S}}(z)\right)}{\left\|z-P_{\bar{S}}(z)\right\|} .
$$

From (2.3) and (2.4), $y^{*} \in B^{*}(0, \alpha) \cap\left(\bigcup_{x \in \bar{S}} N_{\bar{S}}(x)\right)$. From (3.2), there exists $w \in \bar{S}$ such that $y^{*} \in T(w)$. It follows from Lemma 3.3 that $y^{*} \in T\left(P_{\bar{S}}(z)\right)$. Since $T$ is monotone, we have $0 \leq\left\langle z-P_{\bar{S}}(z), w^{*}-y^{*}\right\rangle$, and hence

$$
\begin{aligned}
\left\langle z-P_{\bar{S}}(z), y^{*}\right\rangle & \leq\left\langle z-P_{\bar{S}}(z), w^{*}\right\rangle \\
& \leq\left\|z-P_{\bar{S}}(z)\right\|\left\|w^{*}\right\| .
\end{aligned}
$$

From (2.1) and (3.3), we have that $\alpha \leq\left\|w^{*}\right\|$, and this is a contradiction.

\section{Finite termination of the proximal point algorithm}

In this section, we prove that the proximal point algorithm converges to a solution in a finite number of steps under the condition (3.2). Our results include the results of Ferris [13] and Mangasarian 21] obtained in a finite dimensional setting as special cases. Let $E$ be a smooth, strictly convex and reflexive Banach space and let $T$ be a maximal monotone operator. Then $T$ is maximal if and only if $R(J+r T)=E^{*}$ for all $r>0$; see [25, 4, 30, 28. Thus, we can define, for each $r>0$, a single-valued mapping $J_{r}: E \rightarrow D(T)$ by $J_{r}=(J+r T)^{-1} J$. It is called the resolvent of $T$. We can also define, for each $r>0$, the Yosida approximation of $T$ by $A_{r}=r^{-1}\left(J-J J_{r}\right)$. We know that $\left(J_{r}(x), A_{r}(x)\right) \in G(T)$ for all $r>0$ and $x \in E$. See [3, 4, 30] for details. The resolvents of maximal monotone operators have the following property. 
Lemma 4.1 ([19, Lemma 3.1]). Let $E$ be a smooth, strictly convex and reflexive Banach space, let $T: E \rightarrow 2^{E^{*}}$ be a maximal monotone operator with $T^{-1}(0) \neq \emptyset$, let $r>0$ and let $J_{r}=(J+r T)^{-1} J$. Then

$$
\phi\left(u, J_{r}(x)\right)+\phi\left(J_{r}(x), x\right) \leq \phi(u, x) \text { for all } u \in T^{-1}(0) \text { and } x \in E,
$$

where $\phi: E \times E \rightarrow \mathbb{R}$ is a function defined by

$$
\phi(x, y)=\|x\|^{2}-2\langle x, J(y)\rangle+\|y\|^{2} \text { for all } x, y \in E .
$$

The following lemma was proved in [15].

Proposition 4.2 ([15, Proposition 2]). Let $E$ be a smooth and uniformly convex Banach space and let $\left\{x_{n}\right\}$ and $\left\{y_{n}\right\}$ be sequences in $E$ such that either $\left\{x_{n}\right\}$ or $\left\{y_{n}\right\}$ is bounded. If $\lim _{n \rightarrow \infty} \phi\left(x_{n}, y_{n}\right)=0$, then $\lim _{n \rightarrow \infty}\left\|x_{n}-y_{n}\right\|=0$.

Our first main result is stated as follows.

Theorem 4.3. Let $E$ be a uniformly smooth and uniformly convex Banach space and let $T: E \rightarrow 2^{E^{*}}$ be a maximal monotone and paramonotone operator and let $\left\{x_{n}\right\}$ be a sequence defined as follows: $x_{1} \in E$ and

$$
x_{n+1}=J_{r_{n}}\left(x_{n}\right)(n=1,2, \cdots),
$$

where $\left\{r_{n}\right\} \subset(0, \infty)$ satisfies $\liminf _{n \rightarrow \infty} r_{n}>0$ and $J_{r_{n}}=\left(J+r_{n} T\right)^{-1} J$. If condition (3.2) holds, then $x_{n} \in \bar{S}=T^{-1}(0)$ for all sufficiently large $n$.

Proof. Since $A_{r_{n}}\left(x_{n}\right) \in T\left(J_{r_{n}}\left(x_{n}\right)\right)=T\left(x_{n+1}\right)$, it is sufficient to show that $A_{r_{n}}\left(x_{n}\right)$ $\rightarrow 0$ as $n \rightarrow \infty$. This result has in fact been shown in [16. The proof is retrieved here for the sake of completeness. Let $u \in \bar{S}$. Since Lemma 4.1 implies that, for each $n \in \mathbb{N}$,

$$
\phi\left(u, x_{n+1}\right)+\phi\left(x_{n+1}, x_{n}\right) \leq \phi\left(u, x_{n}\right),
$$

from (4.3), $\lim _{n \rightarrow \infty} \phi\left(u, x_{n}\right)$ exists and further $\lim _{n \rightarrow \infty} \phi\left(x_{n+1}, x_{n}\right)=0$. It follows from Proposition 4.2 that $\lim _{n \rightarrow \infty}\left\|x_{n+1}-x_{n}\right\|=0$. Since $E$ is uniformly smooth, the duality mapping $J$ is uniformly norm-to-norm continuous on each bounded subset of $E$. Thus, we have that

$$
\lim _{n \rightarrow \infty}\left\|J\left(x_{n+1}\right)-J\left(x_{n}\right)\right\|=\lim _{n \rightarrow \infty}\left\|J\left(J_{r_{n}}\left(x_{n}\right)\right)-J\left(x_{n}\right)\right\|=0 .
$$

From (4.4) and $\lim \inf _{n \rightarrow \infty} r_{n}>0$, we have

$$
\left.\lim _{n \rightarrow \infty}\left\|A_{r_{n}}\left(x_{n}\right)\right\|=\lim _{n \rightarrow \infty} \frac{1}{r_{n}} \| J\left(x_{n}\right)-J\left(J_{r_{n}}\left(x_{n}\right)\right)\right) \|=0 .
$$

This implies that there exists $n_{0} \in \mathbb{N}$ such that $\left\|A_{r_{n}}\left(x_{n}\right)\right\|<\alpha$ for all $n \geq n_{0}$. It follows from Lemma 3.5 that $x_{n} \in \bar{S}=T^{-1}(0)$ for all $n \geq n_{0}+1$.

The second theorem of this section states that for any given $x \in E$, the proximal point algorithm has one step termination if $r_{n}$ is chosen to be sufficiently large. The strong convergence result was obtained by Reich [23]. It should be noted that Reich's result does not guarantee the finite termination of the algorithm. Now, we obtain the following finite convergence result.

Theorem 4.4. Let $E$ be a smooth, strictly convex and reflexive Banach space $E$, let $T: E \rightarrow 2^{E^{*}}$ be a maximal monotone and paramonotone operator, and let $\left\{x_{n}\right\}$ be a sequence defined as follows: $x \in E$ and

$$
x_{n}=J_{r_{n}}(x)(n=1,2, \cdots),
$$


where $\left\{r_{n}\right\} \subset(0, \infty)$ satisfies $\lim _{n \rightarrow \infty} r_{n}=\infty$ and $J_{r_{n}}=\left(J+r_{n} T\right)^{-1} J$. If condition (3.2) holds, then $x_{n} \in \bar{S}=T^{-1}(0)$ for all sufficiently large $n$.

Proof. Let $u \in \bar{S}$. Since Lemma 4.1 implies that, for each $n \in \mathbb{N}$,

$$
\phi\left(u, x_{n}\right) \leq \phi\left(u, x_{n}\right)+\phi\left(x_{n}, x\right) \leq \phi(u, x),
$$

and since $(\|a\|-\|b\|)^{2} \leq \phi(a, b)$ for all $a, b \in E$ by (4.1), the sequence $\left\{x_{n}\right\}$ is bounded. Moreover, since (2.1) implies that the sequence $\left\{J\left(x_{n}\right)\right\}$ is also bounded, it follows from (4.5) that

$$
\frac{1}{r_{n}}\left(J(x)-J\left(x_{n}\right)\right) \in T\left(x_{n}\right) .
$$

Therefore, the desired result is obtained from Lemma 3.5, because $\lim _{n \rightarrow \infty} r_{n}=$ $\infty$.

\section{Applications in optimization}

As a direct consequence, we will now describe two iterative schemes for solving the convex minimization problem.

Theorem 5.1. Let $E$ be a uniformly smooth and uniformly convex Banach space and let $f: E \rightarrow \overline{\mathbb{R}}$ be a proper, convex and lower semicontinuous function such that $\partial f^{-1}(0) \neq \emptyset$ and let $\left\{x_{n}\right\}$ be a sequence defined as follows: $x_{1} \in E$ and

$$
x_{n+1}=\underset{y \in E}{\operatorname{argmin}}\left\{f(y)+\frac{1}{2 r_{n}}\|y\|^{2}-\frac{1}{r_{n}}\left\langle y, J\left(x_{n}\right)\right\rangle\right\}(n=1,2, \ldots),
$$

where $\left\{r_{n}\right\} \subset(0, \infty)$ satisfies $\liminf _{n \rightarrow \infty} r_{n}>0$. If the set $\bar{S}=\partial f^{-1}(0)$ is a set of weak sharp minima with modulus $\alpha>0$, i.e.,

$$
f(y) \geq f(u)+\alpha d(y, \bar{S}) \text { for all } u \in \bar{S} \text { and } y \in E,
$$

where $d(y, \bar{S})=\inf _{z \in \bar{S}}\|y-z\|$, then $x_{n} \in \bar{S}$ for all sufficiently large $n$.

Proof. By Rockafellar's theorem [24] and Proposition 2.1, the subdifferential mapping $\partial f: E \rightarrow 2^{E^{*}}$ is maximal monotone and paramonotone. Fix $r>0, z \in E$ and let $J_{r}$ be the resolvent of $\partial f$. Then we have

$$
J(z) \in J\left(J_{r}(z)\right)+r \partial f\left(J_{r}(z)\right)
$$

and hence

$$
0 \in \partial f\left(J_{r}(z)\right)+\frac{1}{r} J\left(J_{r}(z)\right)-\frac{1}{r} J(z)=\partial\left(f+\frac{1}{2 r}\|\cdot\|^{2}-\frac{1}{r} J(z)\right)\left(J_{r}(z)\right) .
$$

Thus we have

$$
J_{r}(z)=\underset{x \in E}{\operatorname{argmin}}\left\{f(x)+\frac{1}{2 r}\|x\|^{2}-\frac{1}{r}\langle x, J(z)\rangle\right\} .
$$

Therefore, $x_{n+1}=J_{r_{n}}\left(x_{n}\right)$ for all $n \in \mathbb{N}$. On the other hand, it has been shown 9 , Theorem 2.3] that condition (5.1) holds if and only if the condition

$$
B^{*}(0, \alpha) \cap\left(\bigcup_{x \in \bar{S}} N_{\bar{S}}(x)\right) \subset \bigcup_{x \in \bar{S}} \partial f(x)
$$

holds. Using Theorem 4.3 $x_{n} \in \bar{S}$ for all sufficiently large $n$. 
Theorem 5.2. Let $E$ be a smooth, strictly convex and reflexive Banach space, let $f: E \rightarrow \overline{\mathbb{R}}$ be a proper, convex and lower semicontinuous function such that $\partial f^{-1}(0) \neq \emptyset$, and let $\left\{x_{n}\right\}$ be a sequence defined as follows: $x \in E$ and

$$
x_{n}=\underset{y \in E}{\operatorname{argmin}}\left\{f(y)+\frac{1}{2 r_{n}}\|y\|^{2}-\frac{1}{r_{n}}\langle y, J(x)\rangle\right\}(n=1,2, \ldots),
$$

where $\left\{r_{n}\right\} \subset(0, \infty)$ satisfies $\lim _{n \rightarrow \infty} r_{n}=\infty$. If the set $\bar{S}=\partial f^{-1}(0)$ is a set of weak sharp minima with modulus $\alpha>0$, then $x_{n} \in \bar{S}$ for all sufficiently large $n$.

Remark 5.3. Theorems 5.1 and 5.2 include the results of Mangasarian 21, Theorem 2.13] and Ferris [13, Theorems 6 and 7] obtained in a finite dimensional setting as special cases.

\section{ACKNowledgments}

The authors are grateful to Professor W. Takahashi of Tokyo Institute of Technology and Professor D. Kuroiwa of Shimane University for their helpful support. The authors also thank an anonymous referee for a careful reading and helpful comments.

\section{REFERENCES}

[1] Y. I. Alber, Metric and generalized projection operators in Banach spaces: Properties and applications. In Theory and Applications of Nonlinear Operators of Accretive and Monotone Type (A. G. Kartsatos, ed.), Lecture Notes in Pure and Appl. Math., 178, Marcel Dekker, New York, 15-50, 1996. MR1386667 (97b:47068)

[2] M. M. Alves and B. F. Svaiter, A new proof for maximal monotonicity of subdifferential operators, J. Convex Anal. 15 (2008), 345-348. MR2422994 (2010b:49032)

[3] J.-P. Aubin and I. Ekeland, Applied Nonlinear Analysis. John Wiley \& Sons, New York, 1984. MR.749753 (87a:58002)

[4] V. Barbu and T. Precupanu, Convexity and Optimization in Banach Spaces, second edition, D. Reidel, Dordrecht, 1986. MR860772 (87k:49045)

[5] H. H. Bauschke and P. L. Combettes, Construction of best Bregman approximations in reflexive Banach spaces, Proc. Amer. Math. Soc. 131 (2003), 3757-3766. MR.1998183(2004f:41040)

[6] H. H. Bauschke, J. V. Burke, F. R. Deutsch, H. S. Hundal and J. D. Vanderwerff, A new proximal point iteration that converges weakly but not in norm, Proc. Amer. Math. Soc. 133 (2005), 1829-1835. MR2120284 (2005i:90042)

[7] H. Brézis and P.-L. Lions, Produits infinis de résolvantes, Israel J. Math. 29 (1978), 329-345. MR 491922(80b:47068)

[8] J. V. Burke and M. C. Ferris, Weak sharp minima in mathematical programming, SIAM J. Control Optim. 31 (1993), 1340-1359. MR1234006 (94e:90108)

[9] J. V. Burke and S. Deng, Weak sharp minima revisited, Part I: Basic theory, Control \& Cybernetics 31 (2002), 439-469. MR.1978735 (2005a:90155)

[10] Y. Censor, A. N. Iusem and S. A. Zenios, An interior point method with Bregman functions for the variational inequality problem with paramonotone operators, Math. Program. 31 (1998), 373-400. MR1617732 (99f:90147)

[11] I. Cioranescu, Geometry of Banach spaces, Duality Mapping and Nonlinear Problems, Kluwer Academic Publishers, Amsterdam, 1990. MR.1079061 (91m:46021)

[12] A. N. Iusem, On some properties of paramonotone operators, J. Convex Anal. 5 (1998), 269-278. MR.1670352 (99m:47081)

[13] M. C. Ferris, Finite termination of the proximal point algorithm, Math. Program. 50 (1991), 359-366. MR.1114237 (92e:90074)

[14] O. Güler, On the convergence of the proximal point algorithm for convex minimization, SIAM J. Control Optim. 29 (1991), 403-419. MR1092735 (92c:90086)

[15] S. Kamimura and W. Takahashi, Strong convergence of a proximal-type algorithm in a Banach space, SIAM J. Optim. 13 (2002), 938-945. MR1972223 (2004c:90096) 
[16] S. Kamimura, F. Kohsaka and W. Takahashi, Weak and strong convergence theorems for maximal monotone operators in a Banach space, Set-Valued Anal. 12 (2004), 417-429. MR2112848 (2006b:90114)

[17] G. Kassay, The proximal point algorithm for reflexive Banach spaces, Studia Univ. Babes Bolyai Math. 30 (1985), 9-17. MR833677(87h:65103)

[18] K. Kido, Strong convergence of resolvents of monotone operators in Banach spaces, Proc. Amer. Math. Soc. 103 (1988), 755-758. MR947652 (89i:47094)

[19] F. Kohsaka and W. Takahashi, Strong convergence of an iterative sequence for maximal monotone operators in a Banach space, Abstr. Appl. Anal. 2004 (2004), 239-249. MR2058504 (2005e:47113)

[20] F. J. Luque, Asymptotic convergence analysis of the proximal point algorithm, SIAM J. Control Optim. 22 (1984), 277-293. MR732428 (85j:90039)

[21] O. L. Mangasarian, Error bounds for nondegenerate monotone linear complementarity problems, Math. Program. 48 (1990), 437-445. MR1078196 (92i:90107)

[22] B. Martinet, Régularisation d'inéquations variationnelles par approximations successives, Rev. Française Informat. Recherche Opérationnelle 4 (1970), 154-158. MR0298899 (45:7948)

[23] S. Reich, Constructive techniques for accretive and monotone operators, Applied Nonlinear Analysis (V. Lakshmikantham, ed.), Academic Press, New York, 1979, pp. 335-345. MR.537545 (80g:47059)

[24] R. T. Rockafellar, On the maximal monotonicity of subdifferential mappings, Pacific J. Math. 33 (1970), 209-216. MR0262827 (41:7432)

[25] R. T. Rockafellar, On the maximality of sums of nonlinear monotone operators, Trans. Amer. Math. Soc. 149 (1970), 75-88. MR0282272 (43:7984)

[26] R. T. Rockafellar, Monotone operators and the proximal point algorithm, SIAM J. Control Optim. 14 (1976), 877-898. MR0410483 (53:14232)

[27] R. T. Rockafellar and R. J.-B. Wets, Variational Analysis, Springer-Verlag, Berlin, 1998. MR1491362 (98m:49001)

[28] S. Simons and C. Zălinescu, A new proof for Rockafellar's characterization of maximal monotone operators, Proc. Amer. Math. Soc. 132 (2004), 2969-2972. MR.2063117 (2005f:47121)

[29] M.V. Solodov and B.F. Svaiter, Forcing strong convergence of proximal point iterations in a Hilbert space, Math. Program. 87 (2000), 189-202. MR 1734665 (2000j:90077)

[30] W. Takahashi, Nonlinear Functional Analysis. Fixed Points Theory and Its Applications, Yokohama Publishers, Yokohama, 2000. MR1864294 (2002k:47001)

[31] N. Xiu and J. Zhang, On finite convergence of proximal point algorithms for variational inequalities, J. Math. Anal. Appl. 312 (2005), 148-158. MR2175211(2006g:49028)

Department of Electronics and Information Systems, Faculty of Systems Sciences and Technology, Akita Prefectural University, 84-4 Ebinokuchi Tsuchiya, Yurihonjo City, AKITA, 015-0055 JAPAN

E-mail address: matsushita@akita-pu.ac.jp

Department of Electronics and Information Systems, Faculty of Systems Sciences and Technology, Akita Prefectural University, 84-4 Ebinokuchi Tsuchiya, Yurihonjo City, AKITA, 015-0055 JAPAN

E-mail address: xuli@akita-pu.ac.jp 